Archive for

Organic Chemistry

Arkivoc 2018, part vii, 110-118

\title{
Synthesis and biological screening of diethyl [N-(thiazol-2-yl)carbamoyl]methylphosphonates
}

Emmanuel O. Olawode, ${ }^{\text {a }}$ Roman Tandlich, ${ }^{\text {a,b }}$ Earl Prinsloo, ${ }^{b, c}$ Michelle Isaacs, ${ }^{c}$ Heinrich Hoppe, ${ }^{b, d}$ Ronnett Seldon, ${ }^{f}$ Digby F. Warner, ${ }^{g}$ Vanessa Steenkamp, ${ }^{h}$ and Perry T. Kaye ${ }^{* b, e}$

${ }^{a}$ Division of Pharmaceutical Chemistry, Faculty of Pharmacy. ${ }^{b}$ Centre for Chemico- and Biomedicinal Research;

${ }^{c}$ Biotechnology Innovation Centre; ${ }^{d}$ Department of Biochemistry and Microbiology; and ${ }^{e}$ Department of Chemistry, Rhodes University, Grahamstown, South Africa. ${ }^{f}$ Drug Discovery and Development Centre (H3-D), Department of Chemistry and ${ }^{g}$ Molecular Mycobacteriology Research Unit, Department of Pathology and Institute of Infectious Disease and Molecular Medicine, University of Cape Town, Cape Town, South Africa.

${ }^{h}$ Phytomedicine Unit, Department of Pharmacology, University of Pretoria, Pretoria, South Africa Email: E.Olawode@ru.ac.za; P.Kaye@ru.ac.za

Received 02-13-2018

Accepted 03-31-2018

Published on line $09-09-2018$

\section{Abstract}

A three-step synthesis, involving condensation of bromomethyl aryl ketones with urea to afford 2aminothiazoles, their chloroacetylation and subsequent solvent-free Arbuzov phosphonation has afforded a series of novel diethyl [ $N$-(thiazol-2-yl)carbamoyl]methylphosphonates 3a-3f in good overall yields; the 4carboxythiazole analogue $\mathbf{3 g}$ was obtained by selective hydrolysis of the corresponding ethyl ester $\mathbf{3 f}$. The phosphonate esters exhibited significant anti-cancer activity ( $\mathrm{nM}$ - low $\mu \mathrm{M} I \mathrm{IC}_{50}$ values) against SH-SY5Y cells and, in one case, $7.6 \mu \mathrm{M}$ MIC90 anti-TB activity against the virulent $M$. tuberculosis $\mathrm{H}_{37} \mathrm{Rv}$ strain; the chloroacetamido precursors all exhibited some antimalarial ( $P f L D H)$ activity, three with $I_{50}$ values in the range $1.0-8.9 \mu \mathrm{M}$.<smiles>[R]c1csc(NC(=O)CCl)n1</smiles>

Keywords: 2-(2-Chloroacetamido)thiazoles , $\mathrm{N}$-(thiazol-2-yl)carbamoyl]methylphosphonates, synthesis, biological activities 


\section{Introduction}

The enzyme,1-deoxy-D-xylulose-5-phosphate reductoisomerase (DXR) regulates a non-mevalonate pathway in the biosynthesis of isoprenoid-derived compounds in Plasmodium falciparum (Pf), but is not found in humans ${ }^{1,2}$. This enzyme has been validated as a target for the development of antimalarial drugs capable of selectively inhibiting reduction of 1-deoxy-D-xylulose-5-phosphate 1 in resistant $P$. falciparum strains ${ }^{3-5}$. The naturally occurring antibiotic, fosmidomycin [3-( $N$-formyl- $N$-hydroxyamino)propylphosphonic acid ${ }^{4,5}$ and its $N$ acetyl derivative, FR900098 $\mathbf{2}^{6,7}$, are known to inhibit PfDXR, and various analogues of these compounds have been prepared. In our own group, research has focussed on the synthesis and antimalarial activity of phosphonated $\mathrm{N}$-aryl- and $\mathrm{N}$-heteroaryl-carboxamides, such as compound $\mathbf{3}^{8,9}$ and, more recently, phosphoramidate analogues ${ }^{6}$ of fosmidomycin.
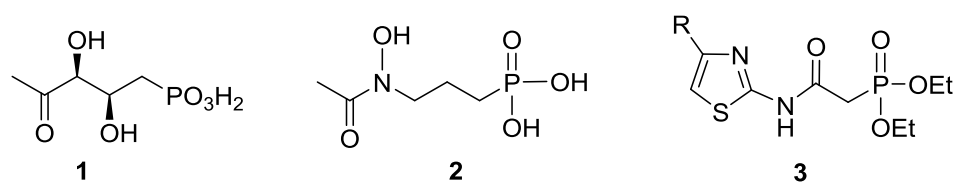

The thiazole scaffold is well represented in medicinal systems. Mjambili et al. have prepared a library of $\mathrm{N}$ aryl substituted 2-(pyridin-2-yl)thiazol-4-amines and explored their anti-TB and antimalarial activity, and compounds containing electronegative para substituents were shown to inhibit $P$. falciparum with submicromolar $\mathrm{IC}_{50}$ values ${ }^{11}$. The anti-cancer potential of certain thiazole-based compounds has been investigated, the most active of which, ethyl 2-[3-(diethylamino)propanamido]thiazole-4-carboxylate, exhibited a $\mathrm{GI}_{50}$ value of $0.08 \mu \mathrm{M}$ against the RPMI-8226 leukemia cell line and broad-spectrum activity against 60 tumour cell lines with a $\mathrm{Gl}_{50}$ value of $38.3 \mu \mathrm{M}^{12,13}$. Jaishree et al. showed that 2-methyl-4trifluoromethylthiazole-5-carboxamides exhibited promising anti-parasitic and insecticidal properties, but without herbicidal effect. ${ }^{14}$

In this communication, we discuss: (i) the synthesis of a series of [N-(thiazol-2$\mathrm{yl})$ carbamoylmethylphosphonate esters $\mathbf{3}$ as fosmidomycin analogues which satisfy the Lipinski "Rule of Five"; and (ii) screening of these compounds and their synthetic precursors for antimalarial, anti-cancer and antituberculosis activity.

\section{Result and Discussion}

The aminothiazole scaffolds 6a-e were obtained using conventional Hantzsch condensation of the $\alpha$-bromo ketones 4a-f with thiourea (Scheme 1). On completion of each of the reactions, the desired product was precipitated out by pouring the reaction mixture into ice-cold water. The known thiazole derivatives $3 a-f^{15-17}$ were thus isolated in excellent yields (93-100\%; Table 1) and subsequently treated with chloroacetyl chloride in the presence of triethylamine in dichloromethane, using a modification of the method reported by Xu et al. ${ }^{18}$ to afford the 2-(2-chloroacetamido)thiazole analogues $7 \mathrm{a}-\mathrm{f}$ in yields ranging from 54 to $100 \%$. Solventfree Arbuzov phosphonation of the 2-(2-chloroacetamido)thiazoles 7a-f was effected by boiling with triethyl phosphite at $110{ }^{\circ} \mathrm{C}$ for $9 \mathrm{~h}$. Excess triethyl phosphite was removed by stirring the crude products with hexane. Column chromatography afforded the desired phophonate esters 3a-e in $68-98 \%$ yield, while treatment of the carbethoxy analogue $\mathbf{3 f}$ with methanolic potassium hydroxide, followed by acidification, permitted selective 
hydrolysis of the carboxlic ester moiety to give diethyl [(4-carboxythiazol-2-yl)carbamoyl]methylphosphonate $\mathbf{3 g}$ in 59\% yield. The phosphonate esters 3a-g are all new and were fully characterised using 1- and 2-D NMR, IR and HRMS methods.

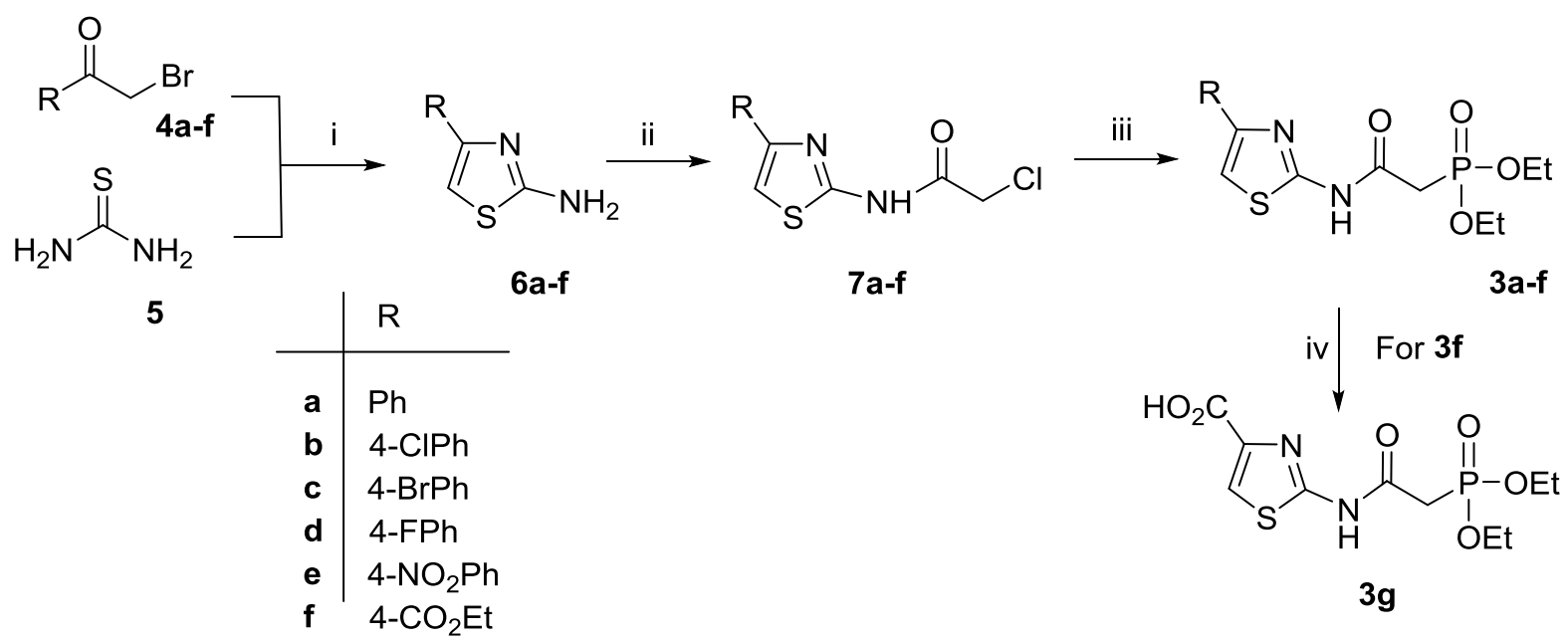

Reagents and conditions: i) EtOH, $70{ }^{\circ} \mathrm{C}, 1 \mathrm{~h}$; ii) Chloroacetyl chloride, $0{ }^{\circ} \mathrm{C}-\mathrm{rt}, 2 \mathrm{~h}$; iii) (EtO) ${ }_{3} \mathrm{P}, 110{ }^{\circ} \mathrm{C}, 9 \mathrm{~h}$; and iv) $\mathrm{KOH}, \mathrm{MeOH}, \mathrm{rt}, 2 \mathrm{~h}$, then $20 \% \mathrm{HCl}$.

\section{Scheme 1}

Table 1. Yields (\%) of the intermediates 6a-f and 7a-f and the diethyl [N-(thiazol-2-yl)carbamoyl]methylphosphonates 3a-g.

\begin{tabular}{|c|c|c|c|c|c|c|c|}
\hline & $R_{\gamma}$ & & & & $\mathrm{R}_{\gamma}$ & & \\
\hline $\mathrm{R}$ & \multicolumn{7}{|c|}{ Yield (\%) } \\
\hline $\mathrm{Ph}$ & $6 a$ & 99 & $7 a$ & 54 & $3 a$ & 98 & \\
\hline$p$-ClPh & $6 b$ & 100 & $7 b$ & 100 & $3 b$ & 89 & \\
\hline$p$-BrPh & $6 c$ & 93 & 7c & 64 & $3 c$ & 68 & \\
\hline$p-\mathrm{FPh}$ & $6 d$ & 94 & $7 d$ & 64 & $3 d$ & 70 & \\
\hline$p-\mathrm{NO}_{2} \mathrm{Ph}$ & $6 e$ & 94 & $7 e$ & 88 & $3 e$ & 70 & \\
\hline COOEt & $6 f$ & 100 & $7 f$ & 70 & $3 f$ & 87 & \\
\hline $\mathrm{COOH}$ & - & - & - & - & $3 g$ & $59^{a}$ & \\
\hline
\end{tabular}

${ }^{\mathrm{a}} \mathbf{3 g}$ obtained from hydrolysis of the carbethoxy precursor $\mathbf{3}$.

It is apparent from the data summarised in Table 2 that, at low concentrations, the phosphonate esters 3ac,e,g exhibit encouragingly effective inhibition of the SH-SY5Y cell line with $\mathrm{IC}_{50}$ values in the nanomolar to very low micromolar range but little, if any, activity against the HeLa cell line ${ }^{19,20}(\geq 95 \%$ viability at $20 \mu \mathrm{M})$, 
thus reflecting clear selectivity against the former cell line. Compounds 7a-f, however, exhibit $15-40 \%$ inhibition of the HeLa cells at $20 \mu \mathrm{M}$.

Table 2. Bioassay results for compounds 6a-f, 7a-f and 3a-g

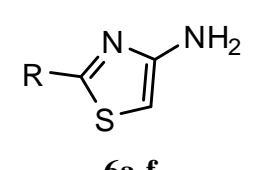

6a-f<smiles>[R]c1nc(NC(=O)CCl)cs1</smiles>

7a-f<smiles>[R]c1nc(NC(=O)CP(=O)(O)OCC)cs1</smiles>

3a-g

\begin{tabular}{|c|c|c|c|c|c|c|c|}
\hline & \multirow[b]{2}{*}{$\mathrm{R}$} & \multicolumn{2}{|c|}{ Anti-cancer } & \multicolumn{2}{|c|}{ Antimalarial } & \multicolumn{2}{|c|}{ Anti-TB } \\
\hline & & $\begin{array}{l}\text { SH-SY5Y } \\
I_{50}(\mu \mathrm{M})\end{array}$ & $\begin{array}{l}\text { HeLa cells } \\
\% \text { Viability }^{a}\end{array}$ & $\begin{array}{l}\text { PfLDH } \\
\text { \% Viability }\end{array}$ & $\mathrm{IC}_{50}(\mu \mathrm{M})$ & $\begin{array}{l}\text { MIC90 } \\
(\mu \mathrm{M})\end{array}$ & $\begin{array}{r}\text { MIC99 } \\
(\mu \mathrm{M})\end{array}$ \\
\hline $6 a$ & $\mathrm{Ph}$ & - & 90 & 85 & - & - & - \\
\hline $6 b$ & $p$-ClPh & - & 98 & 81 & - & - & - \\
\hline $6 c$ & $p-\mathrm{BrPh}$ & - & $\leq 100$ & 88 & - & - & - \\
\hline $6 d$ & $p-\mathrm{FPh}$ & - & $\leq 100$ & 83 & - & - & - \\
\hline $6 e$ & $p-\mathrm{NO}_{2} \mathrm{Ph}$ & - & 95 & 84 & - & - & - \\
\hline $6 f$ & COOEt & - & 75 & 68 & - & - & - \\
\hline $7 a$ & $\mathrm{Ph}$ & - & 80 & 18 & 1.86 & - & - \\
\hline $7 b$ & $p$-ClPh & - & 85 & 40 & - & - & - \\
\hline 7c & $p-\mathrm{BrPh}$ & - & 80 & 32 & - & - & - \\
\hline 7d & $p-\mathrm{FPh}$ & - & 60 & 16 & 8.87 & - & - \\
\hline $7 e$ & $p-\mathrm{NO}_{2} \mathrm{Ph}$ & - & 55 & 14 & 1.04 & - & - \\
\hline $7 f$ & COOEt & - & 65 & 68 & - & - & - \\
\hline $3 a$ & $\mathrm{Ph}$ & 0.87 & 100 & 115 & - & $>20.0$ & $>20.0$ \\
\hline $3 b$ & $p$-ClPh & 0.0018 & 100 & 115 & - & $>20.0$ & $>20.0$ \\
\hline $3 c$ & $p-\mathrm{BrPh}$ & 2.1 & 100 & 102 & - & 7.62 & $>20.0$ \\
\hline $3 d$ & $p-\mathrm{FPh}$ & $>1 \mathrm{mM}$ & 95 & 102 & - & $>20.0$ & $>20.0$ \\
\hline $3 e$ & $p-\mathrm{NO}_{2} \mathrm{Ph}$ & 590 & 100 & 105 & - & $>20.0$ & $>20.0$ \\
\hline $3 f$ & COOEt & $>1 \mathrm{mM}$ & $\leq 100$ & 99 & - & $>20.0$ & $>20.0$ \\
\hline $3 g$ & $\mathrm{COOH}$ & 4.6 & $\leq 100$ & 102 & - & $>20.0$ & $>20.0$ \\
\hline \multicolumn{2}{|c|}{ Controls } & & 100 & & & & \\
\hline \multicolumn{2}{|c|}{ Chloroquine } & & - & - & 0.0143 & - & - \\
\hline \multicolumn{2}{|c|}{ Rifampicin } & & - & - & - & 0.0015 & 0.0016 \\
\hline
\end{tabular}

${ }^{\mathrm{a}}$ At $20 \mu \mathrm{M}$.

The resazurin-based whole-cell $P f L D H$ bioassay was conducted to explore the antimalarial activities of the crucial intermediates and final compounds using $20 \mu \mathrm{M}$ as the cut-off concentration before determining $\mathrm{IC}_{50}$ values for compounds with significant levels of inhibition. The results (Table 1) show that the unsubstituted aminothiazole intermediates 6a-f exhibit low levels of inhibition (66-88\% PfLDH viability), whereas the 2-(2chloroacetamido)thiazole intermediates 7a-e exhibit significant activity at $20 \mu \mathrm{M}$, with $I_{50}$ values of $8.87,1.86$ and $1.04 \mu \mathrm{M}$ for compounds $\mathbf{7 d}, \mathbf{7 a}$ and $\mathbf{7 e}$, respectively. The phosphonate esters 3a-g, which were designed 
primarily as potential inhibitors of $P$. falciparum 1-deoxy-1-D-xylulose-5-phosphate reductoisomerase (PfDXR), unfortunately showed no discernible PfLDH inhibition.

Apart from the para-bromophenyl product 3c, which exhibited an MIC90 value of $7.62 \mu \mathrm{M}$, all other compounds in this series (3) showed little if any inhibitory effect (MIC90 and MIC99 values $\geq 20 \mu \mathrm{M}$ ) on the growth of $M$. tuberculosis $\mathrm{H}_{37} \mathrm{Rv}^{19}$. The relatively high predicted Log $\mathrm{P}$ value of 3.87 for compound $3 c$ may contribute to its absorption across the lipophilic membrane of $M$. tuberculosis $H_{37} R v$, whereas the Log $P$ values for the other compounds $\mathbf{3 a}, \mathbf{3 b}, \mathbf{3 d}$, 3e, $\mathbf{3 f}$ and $\mathbf{3 g}(2.98,3.62,3.18,1.54$ and 1.10, respectively) are all lower.

\section{Conclusions}

The novel diethyl [ $\mathrm{N}$-(thiazol-2-yl)carbamoyl]methylphosphonates (3) were successfully obtained in good overall yields. Although designed as potential antimalarial agents, these compounds failed to exhibit any activity against $P f L D H$, whereas their chloroacetamido precursors (7) all exhibited antimalarial (PfLDH) activity, three with $\mathrm{IC}_{50}$ values in the range $1.0-8.9 \mu \mathrm{M}$. The title compounds did, however, exhibit significant and selective anti-cancer activity (nM - low $\mu \mathrm{M} \mathrm{IC}_{50}$ values) against SH-SY5Y cells and, in one case, 7.6 $\mu \mathrm{M}$ MIC90 anti-TB activity against the virulent $M$. tuberculosis $\mathrm{H}_{37} \mathrm{Rv}$ strain.

\section{Experimental Section}

General. Reagents were supplied by Sigma-Aldrich and used without further purification. Tetrahydrofuran (THF) and methylene chloride were stored over $4 \AA$ molecular sieves. The reaction progress and purity of the compounds were checked by thin layer chromatography (TLC) on pre-coated Merck silica gel G60 $F_{254}$ plates, and viewed under UV light at 254 and $365 \mathrm{~nm}$. Melting points were recorded, uncorrected, using a Reichert hot-plate microscope. Nuclear magnetic resonance (NMR) spectra were recorded on Bruker Avance II 600 $\mathrm{MHz}$, Bruker Avance III HD $400 \mathrm{MHz}$ and Bruker Fourier $300 \mathrm{MHz}$ spectrometers. The NMR chemical shifts are reported in ppm downfield from tetramethylsilane (TMS), and the coupling constants are given in $\mathrm{Hertz}(\mathrm{Hz})$. NMR analyses were carried out in deuterated solvents, such as DMSO- $d_{6}, \mathrm{CDCl}_{3}$, acetone- $d_{6}$ and methanol- $d_{4}$ for standard NMR experiments, and the spectra were calibrated using solvent signals $\left[\delta_{H}: 7.26 \mathrm{ppm}\right.$ for residual $\mathrm{CHCl}_{3}, 2.50 \mathrm{ppm}$ for residual DMSO, $2.05 \mathrm{ppm}$ for residual acetone and 3.31 for residual $\mathrm{MeOH} ; \delta_{C}$ : $77.2 \mathrm{ppm}\left(\mathrm{CDCl}_{3}\right), 39.5 \mathrm{ppm}\left(\mathrm{DMSO}-d_{6}\right), 29.8 \mathrm{ppm}$ (acetone- $\left.d_{6}\right)$ and $\left.49.0 \mathrm{ppm}\left(\mathrm{MeOH}-d_{4}\right)\right]$. Infrared (IR) spectra were obtained using a Perkin Elmer ${ }^{(R)}$ Spectrum 400 Frontier / FT-IR spectrometer. High-resolution mass spectra (HRMS) were recorded on a Waters API Q-TOF Ultima spectrometer (University of Stellenbosch, Stellenbosch, South Africa). NMR spectra for all compounds and the bioassay procedures are provided in the Supporting Information.

The known 2-aminothiazoles (6) were obtained following reported methods. ${ }^{16-18}$ A mixture of thiourea (1.2 $\mathrm{mmol}$ ) and 2-bromoacetophenone $(1 \mathrm{mmol})$ in $\mathrm{EtOH}(2 \mathrm{~mL})$ was stirred at $70{ }^{\circ} \mathrm{C}$ for $1 \mathrm{~h}$. The reaction mixture was cooled to room temperature, poured into ice-cold water, and the resulting precipitate was filtered and dried to give the desired compounds: [6a ( 99\%) as a white solid, mp $150-152{ }^{\circ} \mathrm{C}\left(\mathrm{Lit}^{26}{ }^{26} 149-150{ }^{\circ} \mathrm{C}\right) ; 6 \mathrm{~b}(100 \%)$ as a white solid, $\mathrm{mp} 162-165{ }^{\circ} \mathrm{C}$ (Lit. $\left.{ }^{26} 163-164{ }^{\circ} \mathrm{C}\right) ; 6 \mathrm{c}(0.236 \mathrm{~g}, 93 \%)$ as a white solid, $\mathrm{mp} 179-181{ }^{\circ} \mathrm{C}$ (Lit. ${ }^{26} 180-$ $\left.181{ }^{\circ} \mathrm{C}\right)$; $6 \mathrm{~d}(98 \%)$ as a white solid, $\mathrm{mp} 203.6-204.2{ }^{\circ} \mathrm{C}$ (Lit. ${ }^{28 / 22} 204.0-204.5{ }^{\circ} \mathrm{C}$ ); 6e (94\%) as a bright yellow solid, mp $287-288{ }^{\circ} \mathrm{C}$ (Lit. $\left.{ }^{26,27} 285-286{ }^{\circ} \mathrm{C}\right) ; 6 f(100 \%)$ as a white solid, mp $172-174{ }^{\circ} \mathrm{C}$ (Lit. ${ }^{29-31} 172{ }^{\circ} \mathrm{C}$ ). 
The general procedure for the preparation of the known 2-(2-chloroacetamido)thiazoles (7) involved a modification of the procedure reported by $\mathrm{Xu}$ and collegues ${ }^{18}$. A solution of 2-amino-4-phenylthiazole 6a $(0.530 \mathrm{~g}, 3 \mathrm{mmol})$ and $\mathrm{Et}_{3} \mathrm{~N}(560 \mu \mathrm{L}, 4 \mathrm{mmol})$ in dichloromethane $(15 \mathrm{~mL})$ was cooled to $0-5{ }^{\circ} \mathrm{C}$ in an ice-bath and stirred for $30 \mathrm{~min}$. 2-Chloroacetyl chloride $(578 \mu \mathrm{L}, 6.6 \mathrm{mmol})$ in dry dichloromethane $(1.5 \mathrm{~mL})$ was then added slowly, and the reaction mixture was allowed to warm to room temperature and stirred until the amine was completely consumed ( $c a .1 \mathrm{~h}$, as monitored by TLC). The reaction mixture was diluted with dichloromethane and washed successively with water and saturated brine. The organic layer was dried over anhydrous $\mathrm{Na}_{2} \mathrm{SO}_{4}$, the solvent was removed under reduced pressure and the residue was recrystallised from ethanol to give compound $7 \mathrm{a}(0.413 \mathrm{~g}, 54 \%)$ as light-grey crystals, mp $170-171{ }^{\circ} \mathrm{C}\left(\mathrm{Lit}^{18,20,26} 171-173{ }^{\circ} \mathrm{C}\right)$. The remaining analogues were obtained similarly [7b (100\%) as a brown solid, $\mathrm{mp} 194-195{ }^{\circ} \mathrm{C}$ (Lit. ${ }^{27} \mathrm{mp}$ not cited); 7c (64\%) as a brown solid, $\mathrm{mp} 241-243{ }^{\circ} \mathrm{C}$ (Lit. ${ }^{27} \mathrm{mp}$ not cited); 7d (64\%) as a brown solid, mp $135-137{ }^{\circ} \mathrm{C}$ (Lit. ${ }^{28}$ $\left.135{ }^{\circ} \mathrm{C}\right)$; 7 e $(0.262 \mathrm{~g}, 88 \%)$ as a yellow solid, $\mathrm{mp} 174-176{ }^{\circ} \mathrm{C}\left(\right.$ Lit. $\left.{ }^{29} 175{ }^{\circ} \mathrm{C}\right) ; 7 f(70 \%)$ as a light-brown solid, $\mathrm{mp}$ $213-216^{\circ} \mathrm{C}\left(\right.$ Lit. $\left.^{30} 216^{\circ} \mathrm{C}\right)$.

General procedure for the preparation of the diethyl [ $N$-(thiazol-2-yl)carbamoyl]methylphosphonates (3). A mixture of 2-(2-chloroacetamido)-4-phenylthiazole $7 \mathrm{a}(0.063 \mathrm{~g}, 0.25 \mathrm{mmol})$ and triethyl phosphite $(22 \mu \mathrm{L}, 0.13$ $\mathrm{mmol}$ ) in an oven-dried round-bottomed flask was refluxed (ca. $110^{\circ} \mathrm{C}$ ) for $9 \mathrm{~h}$ under nitrogen ${ }^{1,2}$. The reaction mixture was cooled to room temperature and then stirred 3 times with hexane $(3 \times 700 \mu \mathrm{L}$ for 20 min each, followed each time by decantation of the hexane layer to remove excess triethyl phosphite). The residual solvent was evaporated under reduced pressure, and the crude product was chromatographed [using silica gel; eluting with hexane/EtOAc (4:1)] to yield the diethyl [4-phenylthiazol-2-yl)carbamoyl]methylphosphonate. Diethyl [4-phenylthiazol-2-yl)carbamoyl]methylphosphonate (3a). Brown solid (0.087 g, 98\%), mp 78-80 ${ }^{\circ} \mathrm{C}$; [HRMS: $m / z$ calculated for $\mathrm{C}_{15} \mathrm{H}_{20} \mathrm{~N}_{2} \mathrm{O}_{4} \mathrm{PS}\left(\mathrm{MH}^{+}\right)$355.0881. Found 355.0876]; $v_{\max } / \mathrm{cm}^{-1} 1679$ (C=O) and 3168 $(\mathrm{NH}) ; \delta_{\mathrm{H}}\left(400 \mathrm{MHz} ; \mathrm{CDCl}_{3}\right) 10.96(1 \mathrm{H}, \mathrm{br} \mathrm{s}, \mathrm{NH}), 7.80\left(2 \mathrm{H}, \mathrm{d},{ }^{3} \mathrm{~J} 8 \mathrm{~Hz}, \mathrm{ArH}\right), 7.36-7.29\left(2 \mathrm{H}, \mathrm{m},{ }^{3} \mathrm{~J} 7.1-7.4 \mathrm{~Hz}, \mathrm{ArH}\right)$, $7.01\left(1 \mathrm{H}, \mathrm{s}\right.$, thiazolyl-H), 4.28-4.21 $\left(4 \mathrm{H}, \mathrm{m}, 2 \times \mathrm{OCH}_{2}\right), 3.17\left(2 \mathrm{H}, \mathrm{d},{ }^{2} \mathrm{~J}_{\mathrm{P}, \mathrm{H}} 24 \mathrm{~Hz}, \mathrm{PCH}_{2}\right)$ and $1.39\left(6 \mathrm{H}, \mathrm{t},{ }^{3} \mathrm{~J}_{\mathrm{H}, \mathrm{H}} 8.0 \mathrm{~Hz}\right.$, $\left.2 \times \mathrm{CH}_{3}\right) ; \delta_{\mathrm{C}}\left(100 \mathrm{MHz} \mathrm{CDCl}_{3}\right) 162.4\left(\mathrm{C}=\mathrm{O},{ }^{2}{ }_{\mathrm{C}, \mathrm{P}} 5 \mathrm{~Hz}\right), 157.3,150.2,134.7,128.7,128.0,126.3,107.8$ (ArC and thiazolyl-C), $63.5\left(\mathrm{OCH}_{2},{ }^{2} J_{\mathrm{C}, \mathrm{P}} 6.4 \mathrm{~Hz}\right), 35.9\left(\mathrm{PCH}_{2},{ }^{1} J_{\mathrm{C}, \mathrm{P}} 131 \mathrm{~Hz}\right)$ and $16.5\left(\mathrm{CH}_{3},{ }^{3} J_{\mathrm{C}, \mathrm{P}} 5.9 \mathrm{~Hz}\right)$.

Diethyl $\{\boldsymbol{N}$-[4-(4-chlorophenyl)thiazol-2-yl]carbamoyl\}methylphosphonate (3b). Brown solid (0.087 g, $89 \%)$, mp 86-89 ${ }^{\circ} \mathrm{C}$; [HRMS: $\mathrm{m} / z$ calculated for $\mathrm{C}_{15} \mathrm{H}_{19} \mathrm{ClN}_{2} \mathrm{O}_{4} \mathrm{PS}^{35}\left(\mathrm{MH}^{+}\right) 389.0492$. Found 389.0488]; $\mathrm{v}_{\max } / \mathrm{cm}^{-1} 1680$ $(\mathrm{C}=\mathrm{O})$ and $3169(\mathrm{NH}) ; \delta_{\mathrm{H}}\left(400 \mathrm{MHz} ; \mathrm{CDCl}_{3}\right) 10.99(1 \mathrm{H}, \mathrm{br} \mathrm{s}, \mathrm{NH}), 7.72\left(2 \mathrm{H}, \mathrm{d},{ }^{3} \mathrm{~J}_{\mathrm{H}, \mathrm{H}} 7.8 \mathrm{~Hz}, \operatorname{ArH}\right), 7.36\left(2 \mathrm{H}, \mathrm{d},{ }^{3} \mathrm{~J}_{\mathrm{H}, \mathrm{H}}\right.$ $7.8 \mathrm{~Hz}, \mathrm{ArH}), 6.99\left(1 \mathrm{H}, \mathrm{s}\right.$, thiazolyl-H), $4.27\left(4 \mathrm{H}, \mathrm{m}, 2 \times \mathrm{OCH}_{2}\right), 3.23\left(2 \mathrm{H}, \mathrm{d}^{2}{ }^{2} \mathrm{~J}_{\mathrm{P}, \mathrm{H}} 21 \mathrm{~Hz}, \mathrm{PCH}_{2}\right)$ and $1.40\left(6 \mathrm{H}, \mathrm{t},{ }^{3} \mathrm{~J}_{\mathrm{H}, \mathrm{H}}\right.$ $\left.6.8 \mathrm{~Hz}, 2 \times \mathrm{CH}_{3}\right) ; \delta_{\mathrm{C}}\left(100 \mathrm{MHz} ; \mathrm{CDCl}_{3}\right) 162.5\left(\mathrm{C}=\mathrm{O},{ }^{2} J_{\mathrm{C}, \mathrm{P}} 4.7 \mathrm{~Hz}\right), 157.5,149.1,133.9,133.2,129.0,127.7,108.1$ (ArC and thiazolyl-C), $63.7\left(\mathrm{OCH}_{2},{ }^{2} J_{C, P} 6.5 \mathrm{~Hz}\right), 35.7\left(\mathrm{PCH}_{2},{ }^{1} J_{C, P} 131 \mathrm{~Hz}\right)$ and $16.6\left(\mathrm{CH}_{3},{ }^{3} J_{C, P} 5.8 \mathrm{~Hz}\right)$.

Diethyl $\{\boldsymbol{N}$-[4-(4-bromophenyl)thiazol-2-yl]carbamoyl\}methylphosphonate (3c). Brown solid (0.074 g, 68\%), mp 96-98 ${ }^{\circ} \mathrm{C}$; [HRMS: $m / z$ calculated for $\mathrm{C}_{15} \mathrm{H}_{19} \mathrm{BrN}_{2} \mathrm{O}_{4} \mathrm{PS}^{79}\left(\mathrm{MH}^{+}\right)$432.9987. Found 432.9979]; $\mathrm{v}_{\max } / \mathrm{cm}^{-1} 1680$ $(\mathrm{C}=\mathrm{O})$ and $3160(\mathrm{NH}) ; \delta_{\mathrm{H}}\left(400 \mathrm{MHz} ; \mathrm{CDCl}_{3}\right) 11.02(1 \mathrm{H}, \mathrm{br} \mathrm{s}, \mathrm{NH}), 7.65\left(2 \mathrm{H}, \mathrm{d}^{3}{ }^{3} \mathrm{~J}_{\mathrm{H}, \mathrm{H}} 8.3 \mathrm{~Hz}, \operatorname{ArH}\right), 7.51\left(2 \mathrm{H}, \mathrm{d},{ }^{3} J_{\mathrm{H}, \mathrm{H}}\right.$ $8.3 \mathrm{~Hz}, \mathrm{ArH}), 6.98\left(1 \mathrm{H}, \mathrm{s}\right.$, thiazolyl-H), $4.25\left(4 \mathrm{H}, \mathrm{m}, 2 \times \mathrm{OCH}_{2}\right), 3.22\left(2 \mathrm{H}, \mathrm{d}^{2}{ }^{2} \mathrm{~J}_{\mathrm{P}, \mathrm{H}} 22 \mathrm{~Hz}, \mathrm{PCH}_{2}\right)$ and $1.38\left(6 \mathrm{H}, \mathrm{t},{ }^{3} \mathrm{~J}_{\mathrm{H}, \mathrm{H}}\right.$ $\left.7.0 \mathrm{~Hz}, 2 \times \mathrm{CH}_{3}\right) ; \delta_{\mathrm{C}}\left(100 \mathrm{MHz} ; \mathrm{CDCl}_{3}\right) 162.4\left(\mathrm{C}=\mathrm{O},{ }^{2} \mathrm{~J}_{\mathrm{C}, \mathrm{P}} 4.5 \mathrm{~Hz}\right), 157.5,149.0,133.6,131.8,127.9,121.9,108.1$ (ArC and thiazolyl-C), $63.6\left(\mathrm{OCH}_{2},{ }^{2} J_{\mathrm{C}, \mathrm{P}} 6.5 \mathrm{~Hz}\right), 35.6\left(\mathrm{PCH}_{2},{ }^{1} J_{\mathrm{C}, \mathrm{P}} 130.6 \mathrm{~Hz}\right)$ and $16.5\left(\mathrm{CH}_{3},{ }^{3} \mathrm{~J}_{\mathrm{C}, \mathrm{P}} 6.0 \mathrm{~Hz}\right)$.

Diethyl $\{\boldsymbol{N}$-[4-(4-fluorophenyl)thiazol-2-yl]carbamoyl\}methylphosphonate (3d) Brown solid (0.0651 g, $70 \%)$, mp 72-74 ${ }^{\circ} \mathrm{C}$; [HRMS: $\mathrm{m} / z$ calculated for $\mathrm{C}_{15} \mathrm{H}_{19} \mathrm{FN}_{2} \mathrm{O}_{4} \mathrm{PS}\left(\mathrm{MH}^{+}\right) 373.0787$. Found 373.0786]; $\mathrm{v}_{\max } / \mathrm{cm}^{-1} 1680$ $(\mathrm{C}=\mathrm{O})$ and $3159(\mathrm{NH}) ; \delta_{\mathrm{H}}\left(400 \mathrm{MHz} ; \mathrm{CDCl}_{3}\right) 10.89(1 \mathrm{H}, \mathrm{br} \mathrm{s}, \mathrm{NH}), 7.74\left(2 \mathrm{H}, \mathrm{dd},{ }^{3} J_{\mathrm{H}, \mathrm{H}}=8.3 \mathrm{~Hz},{ }^{4} J_{\mathrm{F}, \mathrm{H}} 5.6, \mathrm{ArH}\right), 7.06$ $\left(2 \mathrm{H}, \mathrm{t},{ }^{3} \mathrm{~J}_{\mathrm{F}, \mathrm{H}}{ }^{3} \mathrm{~J}_{\mathrm{H}, \mathrm{H}} 8.4 \mathrm{~Hz}, \mathrm{ArH}\right), 6.92\left(1 \mathrm{H}, \mathrm{s}\right.$, thiazolyl-H), $4.20\left(4 \mathrm{H}, \mathrm{m}, 2 \times \mathrm{OCH}_{2}\right), 3.18\left(2 \mathrm{H}, \mathrm{d},{ }^{2} \mathrm{~J}_{\mathrm{P}, \mathrm{H}} 21 \mathrm{~Hz} \mathrm{PCH}\right)$ and $1.38\left(6 \mathrm{H}, \mathrm{t},{ }^{3} \mathrm{~J}_{\mathrm{H}, \mathrm{H}} 7.0 \mathrm{~Hz}, 2 \times \mathrm{CH}_{3}\right) ; \delta_{\mathrm{C}}\left(100 \mathrm{MHz} \mathrm{CDCl}_{3}\right) 162.8\left({ }^{1} J_{\mathrm{F}, \mathrm{C}} 247 \mathrm{~Hz}, \mathrm{ArC}\right), 162.3\left(\mathrm{C}=\mathrm{O},{ }^{2} J_{\mathrm{C}, \mathrm{P}} 4.6 \mathrm{~Hz}\right), 157.3$, 
149.3, $130.9\left({ }^{4} J_{F, C} 2.7 \mathrm{~Hz}, \mathrm{ArC}\right), 128.0\left({ }^{3} J_{\mathrm{F}, \mathrm{C}} 8.1 \mathrm{~Hz}, \mathrm{ArC}\right), 115.6\left({ }^{2} J_{\mathrm{F}, \mathrm{C}} 22 \mathrm{~Hz}, \mathrm{ArC}\right), 107.3,63.5\left({ }^{2} J_{\mathrm{C}, \mathrm{P}} 6.5 \mathrm{~Hz}, \mathrm{OCH} 2\right)$, $35.6\left({ }^{1} J_{C, P} 131 \mathrm{~Hz}, \mathrm{PCH}_{2}\right), 16.5\left({ }^{3} J_{\mathrm{C}, \mathrm{P}} 6.0 \mathrm{~Hz}, \mathrm{CH}_{3}\right)$.

Diethyl $\{\boldsymbol{N}$-[4-(4-nitrophenyl)thiazol-2-yl]carbamoyl\}methylphosphonate (3e). Brown solid (0.070 g, $70 \%), \mathrm{mp}$ 88-90 ${ }^{\circ} \mathrm{C}$; [HRMS: $\mathrm{m} / \mathrm{z}$ calculated for $\mathrm{C}_{15} \mathrm{H}_{19} \mathrm{~N}_{3} \mathrm{O}_{6} \mathrm{SP}\left(\mathrm{MH}^{+}\right)$400.0732. Found 400.0717]; $v_{\max } / \mathrm{cm}^{-1} 1683$ (C=O) and $3165(\mathrm{NH}) ; \delta_{\mathrm{H}}\left(400 \mathrm{MHz}\right.$; acetone- $\left.d_{6}\right) 10.84(1 \mathrm{H}, \mathrm{br} \mathrm{s}, \mathrm{NH}), 8.25\left(2 \mathrm{H}, \mathrm{d},{ }^{3} J_{\mathrm{H}, \mathrm{H}} 8.8 \mathrm{~Hz} \mathrm{ArH}\right), 8.10\left(2 \mathrm{H}, \mathrm{d},{ }^{3} J_{\mathrm{H}, \mathrm{H}}\right.$ $8.8 \mathrm{~Hz}, \mathrm{ArH}), 7.66\left(1 \mathrm{H}, \mathrm{s}\right.$, thiazolyl-H), $4.24\left(4 \mathrm{H}, \mathrm{m}, 2 \times \mathrm{OCH}_{2}\right), 3.38\left(2 \mathrm{H}, \mathrm{d}^{2}{ }^{2} \mathrm{~J}_{\mathrm{P}, \mathrm{H}} 22 \mathrm{~Hz}, \mathrm{PCH}_{2}\right)$ and $1.83\left(6 \mathrm{H}, \mathrm{t},{ }^{3} J_{\mathrm{H}, \mathrm{H}}\right.$ $\left.7.1 \mathrm{~Hz}, 2 \times \mathrm{CH}_{3}\right) ; \delta_{\mathrm{C}}\left(100 \mathrm{MHz}\right.$; acetone- $\left.d_{6}\right) 164.2$ (C=O, $\left.{ }^{2} J_{\mathrm{C}, \mathrm{P}} 5.7 \mathrm{~Hz}\right), 159.0,148.2,147.8,141.4,127.5,124.7$, 112.6 (ArC and thiazolyl-C), $63.5\left(\mathrm{OCH}_{2},{ }^{2}{ }_{\mathrm{C}, \mathrm{P}} 6.2 \mathrm{~Hz}\right), 35.9\left(\mathrm{PCH}_{2},{ }^{1} \mathrm{~J}_{\mathrm{C}, \mathrm{P}} 130.1 \mathrm{~Hz}\right)$ and $16.7\left(\mathrm{CH}_{3},{ }^{3} J_{\mathrm{C}, \mathrm{P}} 6.0 \mathrm{~Hz}\right)$.

Diethyl [N-(4-carbethoxythiazol-2-yl)carbamoyl]methylphosphonate (3f). Brown solid (0.154 g, 87\%), mp 74$75{ }^{\circ} \mathrm{C}$; [HRMS: $\mathrm{m} / z$ calculated for $\mathrm{C}_{12} \mathrm{H}_{20} \mathrm{~N}_{2} \mathrm{O}_{6} \mathrm{PS}\left(\mathrm{MH}^{+}\right)$351.0780. Found 351.0773]; $\mathrm{v}_{\max } / \mathrm{cm}^{-1} 1683$ (NC=O), $1721(\mathrm{OC}=\mathrm{O})$ and $3165(\mathrm{NH}) ; \delta_{\mathrm{H}}\left(400 \mathrm{MHz} \mathrm{CDCl}_{3}\right) 11.08(1 \mathrm{H}, \mathrm{br} \mathrm{s}, \mathrm{NH}), 7.78\left(1 \mathrm{H}, \mathrm{s}\right.$, thiazolyl-H), $4.36\left(2 \mathrm{H}, \mathrm{q},{ }^{3} \mathrm{~J}_{\mathrm{H}, \mathrm{H}}\right.$ $\left.7.0 \mathrm{~Hz}, \mathrm{OCH}_{2}\right), 4.32-4.15\left(4 \mathrm{H}, \mathrm{m}, 2 \times \mathrm{OCH}_{2}\right), 3.25\left(2 \mathrm{H}, \mathrm{d},{ }^{2} \mathrm{~J}_{\mathrm{P}, \mathrm{H}} 22 \mathrm{~Hz}, \mathrm{PCH}_{2}\right)$ and 1.45-1.33 (9H, overlapping $\mathrm{m}$, $\left.\mathrm{CH}_{3}\right) ; \delta_{\mathrm{C}}\left(100 \mathrm{MHz} ; \mathrm{CDCl}_{3}\right){ }^{13} \mathrm{C} \mathrm{NMR}\left(101 \mathrm{MHz} \mathrm{CDCl}_{3}\right) \delta 163.4\left(\mathrm{NC}=\mathrm{O}, \mathrm{d}^{2}{ }^{2} \mathrm{~J}_{\mathrm{C}, \mathrm{P}} 5.2 \mathrm{~Hz}\right), 161.7$ (OC=O), 158.0, 142.0, 122.3 (ArC and thiazolyl-C), $63.4\left(\mathrm{OCH}_{2}, \mathrm{~d},{ }^{2} J_{C, P} 6.4 \mathrm{~Hz}\right), 61.5\left(\mathrm{OCH}_{2}\right), 35.7\left(\mathrm{PCH} 2, \mathrm{~d},{ }^{1} \mathrm{~J}_{\mathrm{C}, \mathrm{P}} 132 \mathrm{~Hz}\right), 16.5$ $\left(\mathrm{CH}_{3},{ }^{3} \mathrm{~J}_{\mathrm{C}, \mathrm{P}} 5.9 \mathrm{~Hz}\right)$ and $14.4\left(\mathrm{CH}_{3}\right)$.

Diethyl [N-(4-carboxythiazol-2-yl)carbamoyl]methylphosphonate $\quad \mathbf{3 g})$. A solution of diethyl [(4carbethoxythiazol-2-yl)carbamoyl]methylphosphonate $3 \mathrm{f}(0.088 \mathrm{~g}, 0.25 \mathrm{~mm})$ and $\mathrm{KOH}(0.093 \mathrm{~g}, 0.5 \mathrm{mmol})$ in $\mathrm{MeOH}\left(2 \mathrm{~mL}\right.$ ) was stirred at room temperature for $2 \mathrm{~h} .{ }^{31}$ Addition of $20 \% \mathrm{HCl}(2 \mathrm{~mL})$ gave the desired product $3 \mathrm{~g}$ as a brown viscous oil $(0.0478 \mathrm{~g}, 59 \%)$; [HRMS: $\mathrm{m} / \mathrm{z}$ calculated for $\mathrm{C}_{10} \mathrm{H}_{16} \mathrm{~N}_{2} \mathrm{O}_{6} \mathrm{PS}\left(\mathrm{MH}^{+}\right)$323.0467. Found 323.0456]; $v_{\max } / \mathrm{cm}^{-1} 1697$ (C=O), 2508-3586 (br, COOH) and $3183(\mathrm{NH}) ; \delta_{\mathrm{H}}\left(400 \mathrm{MHz} \mathrm{CDCl}_{3}\right) 11.26$ (br s, $\mathrm{COOH}), 11.02\left(1 \mathrm{H}\right.$, br s, NH), $7.97\left(1 \mathrm{H}, \mathrm{s}\right.$, thiazolyl-H), $4.38\left(4 \mathrm{H}, \mathrm{m}, 2 \times \mathrm{OCH}_{2}\right), 3.43\left(2 \mathrm{H}, \mathrm{d},{ }^{2} \mathrm{JP}_{\mathrm{P}, \mathrm{H}} 21 \mathrm{~Hz}, \mathrm{PCH}_{2}\right)$ and 1.56-1.49 (6H, m, $\left.\mathrm{CH}_{3}\right) ; \delta_{\mathrm{C}}\left(100 \mathrm{MHz} ; \mathrm{CDCl}_{3}\right) 168.2(\mathrm{COOH}), 163.5(\mathrm{C}=\mathrm{O}), 158.1,142.1,122.4$ (thiazolyl-C), 63.5 $\left(\mathrm{OCH}_{2}\right), 35.8\left(\mathrm{CH}_{2}\right)$ and $16.5\left(\mathrm{CH}_{3}\right)$.

\section{Acknowledgements}

The authors thank Rhodes University for financial support and a bursary (E.O.O.) and the South African Medical Research Council (SAMRC) for support with funds from National Treasury under its Economic Competitiveness and Support Package.

\section{References}

1. Rohmer, M. Nat. Prod. Rep. 1999, 16, 565.

https://doi.org/10.1039/a709175c

2. Lichtenthaler, H. K. Biochem. Soc. Trans. 2000, 28, 785.

https://doi.org/10.1042/bst0280785

3. Umeda, T.; Tanaka, N.; Kusakabe, Y.; Nakanishi, M.; Kitade, Y.; Nakamura, K. T. Sci. Rep. 2011, 1, 1. https://doi.org/10.1038/srep00009

4. Adeyemi, C. M.; Faridoon, M.; Isaacs, M.; Mnkandhla, D.; Hoppe, H. C.; Krause, R. W. M.; Kaye P. T. Bioorg. Med. Chem. 2016, 24, 6131.

https://doi.org/10.1016/j.bmc.2016.04.021

5. Deng, L.; Endo, K.; Kato, M.; Cheng, G.; Yajima, S.; Song, Y. ACS Med. Chem. Lett. 2011, 2, 165. 
https://doi.org/10.1021/ml100243r

6. Adeyemi, C. M.; Klein, K.; Isaacs, M.; Mnkandhla, D.; Hoppe, H. C.; Krause, R. W. M.; Kaye, P. T. Tetrahedron, 2017, 73, 1661.

https://doi.org/10.1016/j.tet.2017.01.045

7. Mutorwa, M.; Salisu, S.; Blatch, G. L.; Kenyon, C.; Kaye, P. T. Synth. Commun. 2014, 39, 2723. https://doi.org/10.1080/00397910802663444

8. Bodill, T.; Conibear, A. C.; Blatch, G. L.; Lobb, K. A.; Kaye, P. K. Bioorg. Med. Chem. 2011, $19,1321$. https://doi.org/10.1016/j.bmc.2010.11.062

9. Bodill, T.; Conibear, A. C.; Mutorwa, M. K. M.; Goble, J. L.; Blatch, G. L.; Lobb, K. A.; Klein, R.; Kaye, P. T. Bioorg. Med. Chem. 2013, 21, 4332.

https://doi.org/10.1016/j.bmc.2013.04.076

10. Reichenberg, A.; Wiesner, J.; Weidemeyer, C.; Dreiseidler, E.; Sanderbrand, S.; Altincicek, B.; Beck, E.; Schlitzer, M.; Jomaa, H. Bioorg. Med. Chem. Lett. 2001, 11, 833.

https://doi.org/10.1016/S0960-894X(01)00075-0

11. Mjambili, F.; Njoroge, M.; Naran, K.; De Kock, C.; Smith, P. J.; Mizrahi, V.; Warner, D.; Chibale, K. Bioorg. Med. Chem. Lett. 2014, 24, 560.

https://doi.org/10.1016/j.bmcl.2013.12.022

12. El-Subbagha, H. I.; Abadi, A. H.; Lehmann, J. Arch. Pharm. Med. Chem. 1999, 332: 137.

13. Jaishree, V.; Ramdas, N.; Sachin, J.; Ramesh, B. J. Saudi Chem. Soc. 2012, 16, 371.

https://doi.org/10.1016/j.jscs.2011.02.007

14. Liaras, K.; Geronikaki, a; Glamočlija, J.; Cirić, a; Soković, M. Bioorg. Med. Chem. 2011, $19,3135$. https://doi.org/10.1016/j.bmc.2011.04.007

15. Benaamane, N.; Nedjar-Kolli, B.; Bentarzi, Y.; Hammal, L.; Geronikaki, A.; Eleftheriou, P.; Lagunin, A. Bioorg. Med. Chem. 2008, 16, 3059.

https://doi.org/10.1016/j.bmc.2007.12.033

16. Karegoudar, P.; Karthikeyan, M. S.; Prasad, D. J.; Mahalinga, M.; Holla, B. S.; Kumari, N. S. Eur. J. Med. Chem. 2008, 43, 261.

17. Abedi-Jazini, Z.; Safari, J.; Zarnegar, Z.; Sadeghi, M. Polycycl. Aromat. Compd. 2018, 38, 231. https://doi.org/10.1016/j.ejmech.2007.03.014

18. Xu, Q.; Huang, L.; Liu, J.; Ma, L.; Chen, T.; Chen, J.; Peng, F.; Cao, D.; Yang, Z.; Qiu, N.; Qiu, J.; Wang, G.; Liang, X.; Peng, A.; Xiang, M.; Wei, Y.; Chen, L. Eur. J. Med. Chem. 2012, 52, 70.

https://doi.org/10.1016/i.ejmech.2012.03.006

19. Urcan, E.; Haertel, U.; Styllou, M.; Hickel, R.; Scherthan, H.; Reichl, F. X. Dent. Mater. 2010, $26,51$.

20. Solly, K.; Wang, X.; Xu, X.; Strulovici, B.; Zheng, W. Assay Drug Dev. Technol. 2004, 2, 363.

21. Kocabas, E.; Sarıguney, A. B.; Coskun, A. Heterocycl. 2010, 81(12), 2849-2854.

22. Singh, U. P.; Singh, R. K.; Bhat, H. R.; Subhashchandra, Y. P.; Kumar, V.; Kumawat, M. K.; Gahtori, P. Indian Med. Chem. Res. 2011, 20, 1603. https://doi.org/10.1007/s00044-010-9446-7

23. Rao, K. E.; Bathini, Y.; Lown, J. W. J. Org. Chem. 1990, 55, 728. https://doi.org/10.1021/jo00289a057

24. Plouvier, B.; Houssin, R.; Bailly, C.; Hénichart, J. -P J. Heterocycl. Chem. 1989, 26, 1643. https://doi.org/10.1002/jhet.5570260625

25. Erlenmeyer, H.; Ch Morel, J. Helv. Chim. Acta. 1942, 25, 1073. https://doi.org/10.1002/hlca.19420250529 
26. Papadopoulou, M. V.; Bloomer, W. D.; Lepesheva, G. I.; Rosenzweig, H. S.; Kaiser, M.; Aguilera-Venegas, B.; Wilkinson, S. R.; Chatelain, E.; loset, J. R. J. Med. Chem. 2015, 58, 1307. https://doi.org/10.1021/jm5015742

27. Bhargava, P. N.; Ram, L.; Tripathi, R. J. Indian. Chem. Soc. 1982, 59, 773.

28. Lakhan, R.; Singh, O. M. P. J. Indian Chem. Soc. 1984, 61, 526.

29. Gagiu, F.; Mavrodin, A. Ann. Pharm. Francaises. 1968, 26, 55. 